\title{
Contributors to and impact of residual shunting after device closure of atrial septal defects
}

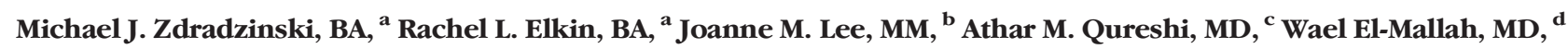
and Richard A. Krasuski, MD ${ }^{\mathrm{e}}$ Cleveland, OH; Houston, Lubbock, TX; and Durbam, NC

Background The prevalence of residual shunt in patients after device closure of atrial septal defect and its impact on long-term outcome has not been previously defined.

Methods From a prospective, single-institution registry of 408 patients, we selected individuals with agitated saline studies performed 1 year after closure. Baseline echocardiographic, invasive hemodynamic, and comorbidity data were compared to identify contributors to residual shunt. Survival was determined by review of the medical records and the Social Security Death Index. Survival analysis according to shunt included construction of Kaplan-Meier curves and Cox proportional hazards modeling.

Results Among 213 analyzed patients, $27 \%$ were men and age at repair was $47 \pm 17$ years. Thirty patients (14\%) had residual shunt at 1 year. Residual shunt was more common with Helex (22\%) and CardioSEAL/STARFlex (40\%) occluder devices than Amplatzer devices (9\%; $P=.005)$. Residual shunts were more common in whites $(79 \%$ vs $46 \%, P=.004)$. At $7.3 \pm 3.3$ years of follow-up, $13(6 \%)$ of patients had died, including $8(5 \%)$ with Amplatzer, $5(25 \%)$ with CardioSEAL/STARFlex, and 0 with Helex devices. Patients with residual shunting had a higher hazard of death (20\% vs $4 \%, P=.001$; hazard ratio 4.95 [1.59-14.90]). In an exploratory multivariable analysis, residual shunting, age, hypertension, coronary artery disease, and diastolic dysfunction were associated with death.

Conclusions Residual shunt after atrial septal defect device closure is common and adversely impacts long-term survival. (Am Heart J 2016;177:1 12-1 19.)

The introduction of percutaneous transcatheter techniques more than 30 years ago revolutionized the correction of secundum atrial septal defects (ASDs). ${ }^{1,2}$ Atrial septal defect repair has been shown to provide multiple benefits, including favorable remodeling of the right heart and a reduction in morbidity and mortality. ${ }^{3-8}$ In addition, functional capacity can improve significantly after closure. $^{9-11}$ For this reason, the American College of Cardiology/American Heart Association 2008 Guidelines for the Management of Adults With Congenital Heart Disease recommend device closure of secundum ASDs in the presence of right heart volume load, evidence of complications such as paradoxical embolism or orthodeoxiaplatypnea, and in the absence of pulmonary hypertension.

From the a Cleveland Clinic Lerner College of Medicine, Case Western Reserve University, Cleveland, $\mathrm{OH},{ }^{b}$ Cardiovascular Medicine, Cleveland Clinic's Heart and Vascular Institute, Cleveland, $\mathrm{OH},{ }^{C}$ Pediatric Cardiology, Texas Children's Hospital/Baylor College of Medicine, Houston, TX, ${ }^{d}$ Cardiovascular Medicine, Texas Tech University Health Sciences Center, Lubbock, TX, and ${ }^{~}$ Cardiology Division, Duke University Health System/ Duke University School of Medicine, Durham, NC.

Lawrence H. Cohn, MD served as guest editor for this article.

Submitted July 19, 2015; accepted March 30, 2016.

Reprint requests: Richard A. Krasuski, MD, Department of Cardiovascular Medicine, Duke University Medical Center, 2301 Erwin Rd, Durham, NC 27710.

E-mail: richard.krasuski@duke.edu

$0002-8703$

(C) 2016 Elsevier Inc. All rights reserved.

http://dx.doi.org/10.1016/i.ahj.2016.03.022
12 Similarly, the 2010 European Society of Cardiology Guidelines recommend closure in cases of significant shunt or after paradoxical embolism. ${ }^{13}$

Percutaneous closure of ASDs appears to be safe and efficacious in comparison to surgical closure. ${ }^{14,15}$ Follow-up studies have demonstrated low rates of periprocedural and short-term complications, including device embolization, erosion, or thrombosis. ${ }^{16-20}$ Other studies have found low rates of medium-to-long-term complications and mortality in pediatric or mixed adult and pediatric populations. ${ }^{21,22}$ However, few studies have examined long-term complications, including the presence of residual shunt and its impact on long-term survival, in the adult population. ${ }^{23}$ Identifying patients at risk for such complications may facilitate improved patient selection and appropriate levels of follow-up to help mitigate this risk. To that end, we present the results of a large, single-center prospective registry of adults who underwent device closure of secundum ASDs.

\section{Methods}

We identified candidates from a prospective, single-center database of adult (age $\geq 18$ years) patients who underwent percutaneous device closure of secundum ASDs from 1999 to 2011. Those with device closure of a patent foramen ovale were excluded from the registry. All patients with adequate 
agitated saline assessment for residual interatrial shunting on transthoracic echocardiography 1 year after closure were included. Interatrial shunting was defined by the presence of at least 3 microbubbles in the left atrium within 3 cycles after opacification of the right atrium, with or without Valsalva maneuver. In addition, patients with inadequate data, those who underwent attempted but aborted device closure, and 1 patient who had subsequent surgical closure were excluded. The study database was approved by the institutional review board of the Cleveland Clinic.

This database includes basic demographic data, procedure details, invasive hemodynamics, and cardiovascular comorbidities at or before the time of the intervention. Procedural details included the date of procedure, device type, and size. We supplemented this with careful review of each patient's electronic medical record as well as the preprocedural echocardiographic imaging and periprocedural transthoracic and intracardiac echocardiographic imaging. Serial echocardiograms after device closure were reviewed by experienced echocardiographers blinded to patient outcome. These included transthoracic studies performed preprocedure and 1 day, 3 months, and 1 year postprocedure. Echocardiographic measures included chamber sizes, presence and severity of valvular regurgitation, right ventricular systolic function, and estimated right ventricular systolic pressures. In addition to calculation of the left ventricular ejection fraction using Simpson's method, right ventricular function (contractility) was graded on a 4-point scale (from 0 to 3 ), where $0=$ normal, $1=$ mildly decreased, $2=$ moderately decreased, and $3=$ severely decreased. Assessment of right ventricle size was similarly reported on a 4-point scale, where $0=$ normal, $1=$ mildly enlarged, 2 = moderately enlarged, and 3 = severely enlarged. The degree of tricuspid regurgitation was determined using color-flow Doppler and assigned a grade from 0 to $4+$, depending on the extent of color flow relative to the right atrial area. Echocardiographic data were further reviewed to identify potential predictors of residual shunting, including the presence of an aneurysmal septum, inadequate aortic rim, or prominent Eustachian valve/Chiari network.

Invasive hemodynamics included right ventricular systolic and pulmonary artery pressures, as well as calculated pulmonary/systemic flow ratio (Qp/Qs). Atrial septal defect size was determined during balloon sizing under fluoroscopy, intracardiac echocardiography, or both during percutaneous closure. When measurements by both methods were available, the average of the 2 values was used for the analysis.

Statistical analysis was performed to determine the prevalence of and the contributors to residual shunt as detected by agitated saline on transthoracic echocardiography at 1 year. Although follow-up echocardiography was also available at other time points, we chose to use residual interatrial shunting at 1 year to account for the possibility of temporary postprocedural shunts that are subsequently sealed during the endothelialization process. The rate of residual shunt by device type was also compared. The 3 categories of devices in this study include the Amplatzer Septal Occluder (St Jude Medical, St Paul, MN), CardioSEAL/ STARFlex Septal Occluder, and the Helex Septal Occluder (Gore Medical, Flagstaff, AR). The CardioSEAL and STARFlex occluders (NMT Medical, Boston, MA) were combined into one group due to their structural similarities, consistent with prior studies. ${ }^{24-27}$

Data from agitated saline studies were available postprocedure and at 3-month and 1-year follow-up. As a secondary analysis to determine the time course of residual shunt, the rates of detected interatrial shunting were compared at each time point using the McNemar test.

We investigated contributors to all-cause death in the entire cohort. Survival data were initially collected from the institutional electronic medical record. We identified the patients' statuses (alive or deceased) at their last known contact with our institution. We then augmented these data with information from the US Social Security Death Index which, at the time of our query, covered all known deaths up to March 1, 2014 (accessed November 2014). A Kaplan-Meier curve was constructed and unadjusted survival was compared using the log-rank test. Stratification was based on the presence of a residual shunt by agitated saline contrast during 1-year follow-up echocardiography. Survival analysis was performed from the time of device closure. Available data on long-term complications were abstracted from our institution's electronic medical record system to assess the prevalence of complications among those with and without residual shunt, as well as the contribution of those complications to patient mortality.

In an exploratory assessment for confounding, hazard ratios with 95\% CIs were calculated using Cox proportional hazards modeling in the whole-cohort mortality analysis. Covariates were selected a priori and included age, gender, ASD balloon size, and the presence of cardiovascular comorbidities. Presence of residual shunt at 1 year was forced into all models tested. Because only 13 deaths occurred in the entire cohort, the exploratory models were constructed with only 2 covariates.

Survival analysis was repeated on the subgroup of those receiving the Amplatzer Septal Occluder device. Kaplan-Meier estimates were again stratified for residual shunting at 1 year. An unadjusted hazard ratio was calculated, but multivariable analysis was not performed due to the limited number of events in the subgroup ( 8 total). We did not perform subgroup analysis for the other device types because no patients with Helex devices died in this study, and the number of patients receiving the CardioSEAL/STARFlex was small and the devices are no longer commercially available.

Baseline demographic, echocardiographic, hemodynamic, and comorbidity data were described using mean and SD or median and interquartile range, as appropriate. Unadjusted 
Table I. Baseline characteristics

\begin{tabular}{|c|c|c|c|c|c|}
\hline & & Entire cohort & Residual shunt & No shunt & $P$ \\
\hline & $\mathrm{n}$ & 213 & 30 & 183 & \\
\hline & Male & $27 \%$ & $37 \%$ & $26 \%$ & .268 \\
\hline & Age (y) & $46.7 \pm 16.6$ & $50.3 \pm 16.4$ & $46.1 \pm 16.6$ & .202 \\
\hline & White & $51 \%$ & $79 \%$ & $46 \%$ & .004 \\
\hline & Black & $45 \%$ & $17 \%$ & $49 \%$ & \\
\hline & Other & $4 \%$ & $3 \%$ & $4 \%$ & \\
\hline & $\mathrm{BMI}\left(\mathrm{kg} / \mathrm{m}^{2}\right)$ & $26.9 \pm 6.8$ & $29.2 \pm 6.9$ & $26.6 \pm 6.7$ & .058 \\
\hline & Diabetes & $3 \%$ & $3 \%$ & $3 \%$ & 1.000 \\
\hline & Hyperlipidemia & $24 \%$ & $20 \%$ & $25 \%$ & .651 \\
\hline & Hypertension & $25 \%$ & $20 \%$ & $26 \%$ & .650 \\
\hline & Coronary artery disease & $9 \%$ & $17 \%$ & $8 \%$ & .171 \\
\hline & Atrial fibrillation & $12 \%$ & $20 \%$ & $11 \%$ & .223 \\
\hline & Chronic kidney disease & $0 \%$ & $0 \%$ & $0 \%$ & \\
\hline & COPD/chronic bronchitis & $1 \%$ & $7 \%$ & $1 \%$ & .053 \\
\hline \multirow[t]{9}{*}{ Echocardiographic data } & Echocardiography follow-up (d) & $340.9 \pm 177.3$ & $414.3 \pm 275.1$ & $327.7 \pm 153.9$ & .102 \\
\hline & Right atrial dilation & $56 \%$ & $74 \%$ & $54 \%$ & .139 \\
\hline & Left atrial dilation & $27 \%$ & $32 \%$ & $27 \%$ & .784 \\
\hline & Right ventricular dilation & $81 \%$ & $93 \%$ & $79 \%$ & .118 \\
\hline & Decreased right ventricular systolic function & $23 \%$ & $25 \%$ & $23 \%$ & .782 \\
\hline & Left ventricular diastolic dysfunction & $13 \%$ & $13 \%$ & $13 \%$ & 1.000 \\
\hline & $>$ Mild mitral regurgitation & $6 \%$ & $3 \%$ & $6 \%$ & 1.000 \\
\hline & $>$ Mild tricuspid regurgitation & $43 \%$ & $42 \%$ & $43 \%$ & 1.000 \\
\hline & Right ventricular systolic pressure (mm Hg) & $36.6 \pm 13.1$ & $34.6 \pm 12.6$ & $37.1 \pm 13.2$ & .502 \\
\hline \multirow{5}{*}{ Catheterization data } & $Q p / Q s$ & $1.9 \pm 0.7$ & $1.9 \pm 0.8$ & $1.9 \pm 0.7$ & .777 \\
\hline & Pulmonary artery mean pressure $(\mathrm{mm} \mathrm{Hg})$ & $18.9 \pm 7.2$ & $17.9 \pm 6.3$ & $19.0 \pm 7.4$ & .597 \\
\hline & Right ventricular systolic pressure $(\mathrm{mm} \mathrm{Hg})$ & $33.3 \pm 12.2$ & $33.8 \pm 9.0$ & $33.2 \pm 12.7$ & .860 \\
\hline & Right ventricular diastolic pressure $(\mathrm{mm} \mathrm{Hg})$ & $5.3 \pm 4.8$ & $5.2 \pm 4.6$ & $5.3 \pm 4.8$ & .917 \\
\hline & ASD size (mm) & $20.1 \pm 6.2$ & $21.7 \pm 6.5$ & $19.8 \pm 6.2$ & .147 \\
\hline \multirow[t]{3}{*}{ Interatrial septal findings } & Aneurysmal interatrial septum & $11 \%$ & $13 \%$ & $11 \%$ & .755 \\
\hline & Thin/absent aortic rim & $1 \%$ & $0 \%$ & $2 \%$ & 1.000 \\
\hline & Prominent Eustachian valve/Chiari network & $4 \%$ & $0 \%$ & $4 \%$ & .604 \\
\hline \multirow[t]{3}{*}{ Device type } & Amplatzer & $73 \%$ & $47 \%$ & $78 \%$ & \\
\hline & CardioSEAL/STARFlex & $9 \%$ & $27 \%$ & $7 \%$ & \\
\hline & Helex & $17 \%$ & $27 \%$ & $15 \%$ & .005 \\
\hline
\end{tabular}

comparison statistics were performed using the $t$ test and Fisher exact test. Analysis involving independent categorical variables with $>2$ values was performed using Pearson $\chi^{2}$ tests. During analysis to determine the time course of residual shunt, the incidences of detected interatrial shunting were compared at each time point using the McNemar test. For all tests, $P<.05$ was considered to be statistically significant. Data analysis was performed using JMP Pro software (SAS Institute, Inc, Cary, NC) version 10.0.2.

No extramural funding was used to support this work. The authors are solely responsible for the design and conduct of this study, all study analyses, the drafting and editing of the manuscript, and its final contents.

\section{Results}

Of the 408 patients in the registry, 213 with 1-year follow-up echocardiograms and adequate agitated saline studies at this time were included in the study. Those excluded from analysis had similar demographics and mortality to those included (Appendix Table 1). Pulmonary pressures were slightly higher and diabetes was more prevalent in the excluded group. The excluded group had more Amplatzers and fewer Helex devices inserted than did the included group.

Within the final cohort, 30 (14\%) had residual shunting on echocardiography at the 1-year follow-up. Amplatzer devices were used in 157 (74\%) of patients, Helex in 36 (17\%), and CardioSEAL/STARFlex in 20 (9\%). The population was $27 \%$ male with a mean age of $46.7 \pm 16.6$ years. There were no differences in age, gender, or the prevalence of cardiovascular comorbidities among patients with and without residual shunt, although chronic obstructive pulmonary disease (COPD)/chronic bronchitis was almost significantly more prevalent in those with residual shunts (Table I). There was a significant difference in the racial composition, with more whites and fewer African Americans having residual shunts $(P=.004)$. Patients with residual shunt also trended toward having higher body mass index (BMI; $29.2 \pm 6.9$ vs $\left.26.6 \pm 6.7 \mathrm{~kg} / \mathrm{m}^{2}, P=.058\right)$.

Baseline echocardiography demonstrated no difference in prevalence of right ventricular systolic dysfunction or the 


\section{Figure 1}

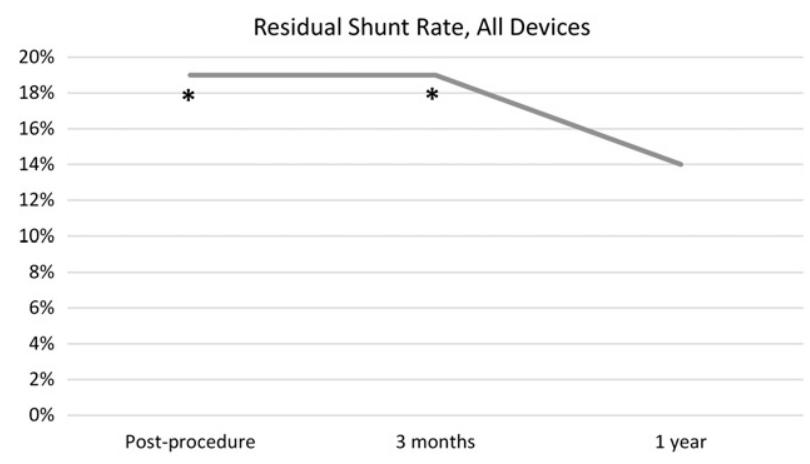

Residual shunt rate over time. Incidence of residual shunt at follow-up in all devices. The overall rate of residual shunt decreased at 1 year compared with postprocedure and 3-month follow-up echocardiograms. ${ }^{*} P<.05$ compared with 1-year time point using the $\mathrm{McNemar}$ test.

presence of greater than mild tricuspid or mitral regurgitation at baseline (Table I). The prevalence of cardiac chamber dilatation was similar in both groups, as was the estimated right ventricular systolic pressure, which was found to be within the normal range for both groups and was consistent with the normal pulmonary artery and right ventricular pressures seen during cardiac catheterization. The calculated Qp/Qs ratio was $1.9 \pm 0.7$ for the combined cohort, with no baseline difference among those with or without residual shunt. There were no differences in ASD size between the 2 groups, nor were there differences in the prevalence of aneurysmal septum, inadequate aortic rim, or prominent Eustachian valve/Chiari networks. Invasive hemodynamics data were available in $59 \%$ of the cohort, but ASD size was assessed in all patients.

Residual shunt was found to significantly differ based on the type of device, with a higher proportion of CardioSEAL/ STARFlex and Helex devices having shunt at 1 year. Those with Amplatzer devices had the lowest residual shunt rate (9\%), followed by Helex (22\%) and CardioSEAL/STARFlex (40\%). The Amplatzer had a significantly lower residual shunt rate than did the other 2 devices $(P<.05$ in paired $t$ test comparisons).

The residual shunt prevalence was $19 \%$ postprocedure and at 3-month follow-up echocardiograms, but fell significantly to $14 \%$ by 1 year $(P=.016)$ (Figure 1$)$.

Six percent of the cohort died during the $7.3 \pm 3.3$ years of follow-up (Table II). The Amplatzer group experienced 8 deaths (5\% of the cohort), the CardioSEAL/STARFlex group experienced 5 deaths (25\%), and the Helex group experienced no deaths. Six (20\%) of those with residual shunts and 7 (4\%) without shunts died during follow-up $(P=.004)$. The presence of a residual shunt at 1 year was associated with a higher hazard of mortality (log-rank $P=.001$ ) (Figure 2).
Table II. Survival data of study cohort

\begin{tabular}{|c|c|c|c|c|}
\hline & $\begin{array}{l}\text { Entire } \\
\text { cohort }\end{array}$ & $\begin{array}{c}\text { Residual } \\
\text { shunt }\end{array}$ & $\begin{array}{l}\text { No } \\
\text { shunt }\end{array}$ & $P$ \\
\hline \multicolumn{5}{|l|}{ All device types } \\
\hline Survival follow-up (y), mean & $8.3 \pm 3.3$ & $9.0 \pm 3.6$ & $8.2 \pm 3.1$ & .630 \\
\hline Deaths & $13(6 \%)$ & $6(20 \%)$ & $7(4 \%)$ & .004 \\
\hline \multicolumn{5}{|l|}{ Amplatzer subgroup } \\
\hline Survival follow-up (y), mean & $7.8 \pm 2.7$ & $7.6 \pm 2.4$ & $7.8 \pm 2.7$ & .753 \\
\hline Deaths & $8(5 \%)$ & $3(21 \%)$ & $5(4 \%)$ & .024 \\
\hline
\end{tabular}

There were no differences in postprocedure complications between those with and without residual shunting (Table III). Patients who died had a significantly higher rate of congestive heart failure diagnosed after their catheterization.

Results of the exploratory Cox proportional hazards analyses are summarized in Table IV. The unadjusted hazard ratio was 4.95 (95\% CI 1.59-14.90) for presence of residual shunt. Age, hypertension, coronary artery disease, and baseline diastolic dysfunction were also significantly associated with mortality when paired with presence of residual shunt, although residual shunt maintained significance in all models. We were unable to assess the impact of diabetes on the hazard of mortality, as no patients with diabetes in this cohort died.

Survival was further examined in the Amplatzer subgroup. Follow-up data were available in the Amplatzer subgroup for an average of $7.8 \pm 2.7$ years after device closure, with no difference between those with and without residual shunt (Table II). During this period, 3 (21\%) with residual shunts and 5 (4\%) without residual shunts died $(P=.024)$. The survival of patients with residual shunts was significantly worse than that of patients without residual shunts at 1 year (log-rank $P=.003$ ) (Figure 3), with an unadjusted hazard ratio of 6.60 (95\% CI 1.35-27.00, $P=.023$ ). Because of the limited number of events in the Amplatzer subgroup, multivariable analysis could not be performed.

\section{Discussion}

\section{Summary of results}

We report the largest single-center study examining predictors of residual shunt and its effect on mortality in adults with percutaneous device closures of ASD. Our database, which included serial echocardiographic follow-up, demonstrated that the prevalence of residual shunting in the adult population after closure is quite significant (14\% 1 year after the procedure). CardioSEAL/ STARFlex and Helex devices were more likely to result in residual shunt, whereas those who received the Amplatzer device experienced a residual shunt rate of only $9 \%$. Device type was the only modifiable predictor of residual shunt. Race also appears to be a predictor, with white patients more likely to experience residual shunt postprocedure. This may reflect differences in the 
Figure 2

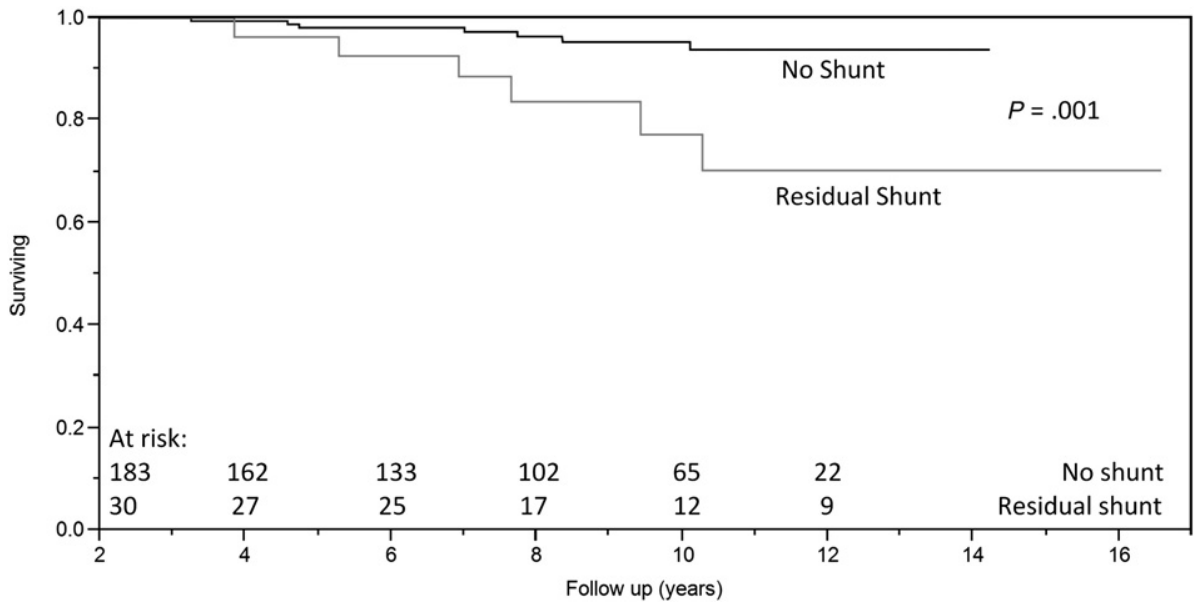

Kaplan-Meier estimate for all devices by presence of residual shunt. Kaplan-Meier curves by presence of residual shunt in all devices. The presence of residual shunt is associated with a higher hazard of mortality (log-rank $P=.001$ ).

Table III. Postprocedure complications

\begin{tabular}{|c|c|c|c|}
\hline & \multicolumn{3}{|c|}{ By presence of residual shunt } \\
\hline & Residual shunt & No shunt & $P$ \\
\hline Stroke/Transient ischemic attack & $0(0 \%)$ & $5(3 \%)$ & 1.000 \\
\hline Congestive heart failure & $2(7 \%)$ & $4(2 \%)$ & .201 \\
\hline New arrhythmia & $1(3 \%)$ & $16(9 \%)$ & .477 \\
\hline Device fracture & $0(0 \%)$ & $1(1 \%)$ & 1.000 \\
\hline \multirow[t]{3}{*}{ Device dislodgement } & $0(0 \%)$ & $1(1 \%)$ & 1.000 \\
\hline & \multicolumn{3}{|c|}{ By mortality } \\
\hline & Death & No death & $P$ \\
\hline Stroke/Transient ischemic attack & $1(8 \%)$ & $4(2 \%)$ & .272 \\
\hline Congestive heart failure & $4(31 \%)$ & $2(1 \%)$ & $<.001$ \\
\hline New arrhythmia & $1(8 \%)$ & $16(8 \%)$ & 1.000 \\
\hline Device fracture & $0(0 \%)$ & $1(1 \%)$ & 1.000 \\
\hline Device dislodgement & $0(0 \%)$ & $1(1 \%)$ & 1.000 \\
\hline
\end{tabular}

intensity of follow-up care, although further exploration is needed to determine the underlying cause of the racial influence on outcomes. Higher BMI trended toward a greater likelihood for residual shunt. Interestingly, baseline echocardiographic data including chamber dilatation did not predict residual shunt, nor did invasive hemodynamics data including shunt ratio, right heart pressures, or ASD size.

Residual shunt declined significantly from the postprocedure and 3-month follow-up echocardiogram to the 1-year follow-up echocardiogram. This suggests that many shunts may close or become undetectable over time, likely due to endothelialization of the device.

Long-term survival in this cohort was good, with a mortality rate of $6 \%$ at 8.3 years of follow-up. Residual shunt was significantly associated with decreased
Table IV. Results of exploratory Cox proportional hazard analyses

\begin{tabular}{lll} 
& Hazard ratio (95\% Cl) & $P$ \\
\hline Shunt & $4.95(1.59-14.90)$ & .007 \\
\hline Shunt & $3.43(1.10-10.37)$ & .035 \\
Age (per year) & $1.11(1.06-1.18)$ & $<.001$ \\
\hline Shunt & $4.89(1.55-14.98)$ & .008 \\
Male & $1.04(0.28-3.27)$ & .948 \\
\hline Shunt & $4.67(1.50-14.07)$ & .009 \\
ASD size (per mm) & $1.15(0.09-11.37)$ & .908 \\
\hline Shunt & $6.26(1.97-19.29)$ & .003 \\
Hypertension & $4.47(1.40-13.85)$ & .013 \\
\hline Shunt & $5.10(1.64-15.40)$ & .006 \\
Hyperlipidemia & $1.99(0.54-6.14)$ & .277 \\
\hline Shunt & $4.27(1.36-13.03)$ & .014 \\
Coronary artery disease & $7.13(1.80-25.02)$ & .007 \\
\hline Shunt & $4.72(1.47-14.64)$ & .011 \\
Atrial fibrillation & $1.29(0.28-4.41)$ & .712 \\
\hline Shunt & $3.86(1.14-12.29)$ & .031 \\
COPD/chronic bronchitis & $6.35(0.92-27.44)$ & .059 \\
\hline Shunt & $4.37(1.40-13.24)$ & .013 \\
Diastolic dysfunction & $6.25(2.00-18.91)$ & .002 \\
\hline
\end{tabular}

survival, with an unadjusted hazard ratio of 4.95 . The residual shunt population was similar to those without shunts with respect to cardiovascular comorbidities and baseline cardiac structure, function, and hemodynamics. Long-term complications were uncommon, and only 


\section{Figure 3}

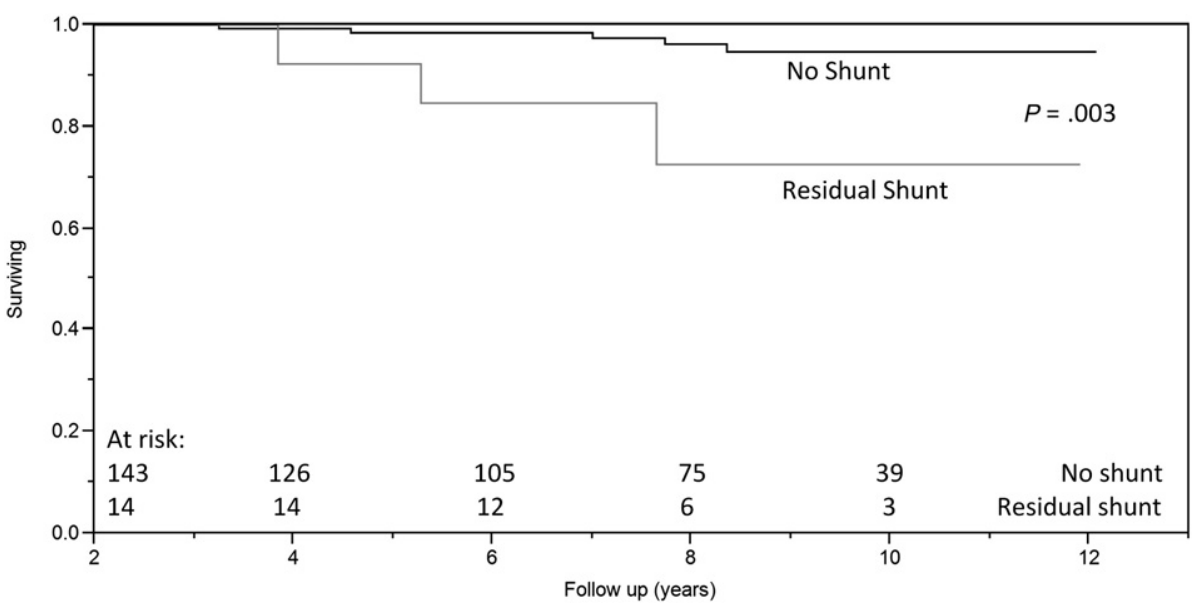

Kaplan-Meier estimate for Amplatzer Septal Occluder by presence of residual shunt. Kaplan-Meier curves by presence of residual shunt in Amplatzer Septal Occluders. The presence of residual shunt remains associated with poorer survival in this cohort (log-rank $P=.003$ ).

new-onset congestive heart failure was more prevalent in patients who died.

The presence of residual shunt remained significant in all exploratory Cox proportional hazards modeling, although age and several cardiovascular comorbidities were also predictive of poorer survival. It is possible that residual shunting indicates a poorly seated device with the potential for erosion or embolization, but further investigation will be needed to determine the cause of death in those with residual shunting.

Interestingly, $100 \%$ of patients with a Helex device survived in the follow-up period, despite having a higher residual shunt rate than those with the Amplatzer. Within the Amplatzer subgroup, the long-term survival rate was 95\%. The unadjusted hazard ratio demonstrated a 6.6 times greater risk of death due to residual shunt in those with Amplatzer.

The population's baseline echocardiographic findings were not surprising. Patients with and without shunt had similar degrees of right and left atrial dilatation and right ventricular dilatation or dysfunction. $43 \%$ of patients had greater than mild tricuspid regurgitation. Right ventricular pressure was normal by invasive hemodynamics and echocardiographic estimation. No structural predictors of residual shunting were identified. The population's average shunt magnitude fell within criteria for closure, with a Qp/Qs of 1.9.

\section{Comparison to current literature}

We report a $14 \%$ residual shunt rate in percutaneous device closure, with CardioSEAL/STARFlex and Helex devices at higher risk for developing residual shunt. This rate correlates well to the experiences of Abdi et al, ${ }^{28}$ who found residual shunts in $13.7 \%$ of cases at 2 centers.
Others have found residual shunt rates ranging from $4 \%$ to $48 \%$, although these studies consisted of a mixed population of adults and pediatric patients. ${ }^{21,29-32}$ Others have also reported significantly higher residual shunt rates with CardioSEAL/STARFlex devices compared with the Amplatzer device, consistent with our results. ${ }^{24,33}$

Our center experienced a low long-term mortality rate of $6 \%$ overall. Several other studies have examined mortality after device closure of ASDs. In their comparison of transcatheter and surgical ASD closure outcomes in adults, Kotowycz et al $^{34}$ demonstrated a $5.3 \%$ mortality rate at 5 years in the percutaneous closure arm. In a 10-year follow-up study of transcutaneous and surgical closure patients, Kutty et al ${ }^{22}$ reported $3 \%$ mortality. It should be noted that $43 \%$ of the population was $<20$ years of age in this study, although the authors did not report the average age. Other short- to medium-term studies have reported no mortality, although many of these included pediatric or mixed populations. ${ }^{15,18,20,35}$ Atrial septal defect closure appears to be safe with a low mortality rate, even at longer follow-up in the adult population.

\section{Recommendations}

Our data suggest that the presence of any residual shunting is associated with higher long-term mortality in adults with percutaneous ASD closure using the Amplatzer device, but not with the Helex. Although the underlying mechanism of this association is unclear, it is possible that patients with residual shunting after Amplatzer insertion may have had an improperly seated device that eventually eroded or resulted in an embolic event, contributing to mortality.

After device closure of ASD, many patients are only followed up with transthoracic echocardiography and 
color flow Doppler interrogation to evaluate for residual shunting. Based on our findings, we recommend following up patients with agitated saline injections, as well. We suggest screening for residual shunt using transthoracic echocardiography during at least 2 follow-up visits, with one screening occurring at least 1-year after device closure. This ensures that the endothelialization process has completed, and that residual shunt is likely to remain in the absence of further intervention. Patients with a residual shunt should be evaluated carefully for their risk of complications, including heart failure, increasing pulmonary arterial pressures, arrhythmia, device thrombosis, erosion, or embolization.

\section{Limitations}

Although the initial registry was collected prospectively, limited follow-up data were available regarding cause of death. One hundred ninety-five patients were excluded from the analysis due to lack of follow-up agitated saline testing. These patients were similar to those included in their demographics and mortality. However, the excluded group had a higher prevalence of diabetes and Amplatzer device use, as well as slightly higher pulmonary pressures compared with those included in the analysis.

Invasive hemodynamics data (excluding ASD size) were available in $59 \%$ of the cohort, limiting our ability to detect differences in cardiac pressures. The presence of residual shunt remained a significant risk factor for death when paired with other known risk factors, but future studies will be required to better explore the mechanisms of death in this population. Second, although it is reassuring that long-term mortality remains low in the ASD population after closure, the low event rate also limited the power for multivariable survival analyses. Our Cox proportional hazards models thus are presented as exploratory analyses. In addition, the test used to detect presence of residual shunt-agitated saline by echocardiography - has limited sensitivity and is often dependent on patient effort during the Valsalva maneuver, in our experience. This could result in some missed residual shunts within the group, although the agitated saline test is the most commonly used method for postprocedure surveillance. Nevertheless, this study contributes important information to the knowledge base of device closure, and we suggest that the presence of residual shunt be evaluated carefully in this population.

\section{Conclusion}

In patients who undergo percutaneous device closure of ASDs, residual shunt is common. Race and device type appear to predict residual shunt at 1 year, with the Helex and CardioSEAL/STARFlex devices having higher rates of residual shunting. The 7-year mortality rate is low in adults after percutaneous device closure, and no mortality was found in the Helex cohort. Residual shunts are associated with poorer survival in the entire cohort and in the Amplatzer subgroup. Patients should be monitored for residual shunting for at least 1 year after device closure, and those with shunting should be closely followed up. Future studies should examine the causes and contributors to mortality in patients with residual shunts.

Supplementary data to this article can be found online at http://dx.doi.org/10.1016/j.ahj.2016.03.022.

\section{Disclosures}

Drs Krasuski and Qureshi have served as investigators for studies using the Amplatzer (St Jude Medical) and Helex devices (Gore Medical). Dr Krasuski has also served as an investigator for studies using the Cardioseal/Starflex device (NMT Medical). The other authors note no conflicts.

\section{References}

1. King TD, Thompson SL, Steiner C, et al. Secundum atrial septal defect. Nonoperative closure during cardiac catheterization. JAMA 1976;235(23):2506-9.

2. Krasuski RA. When and how to fix a "hole in the heart": approach to ASD and PFO. Cleve Clin J Med 2007;74(2):137-47

3. Kort HW, Balzer DT, Johnson MC. Resolution of right heart enlargement after closure of secundum atrial septal defect with transcatheter technique. J Am Coll Cardiol 2001;38(5):1528-32.

4. Takaya $Y$, Taniguchi $M$, Akagi $T$, et al. Long-term effects of transcatheter closure of atrial septal defect on cardiac remodeling and exercise capacity in patients older than 40 years with a reduction in cardiopulmonary function. J Intervent Cardiol 2013;26(2):195-9.

5. Murphy JG, Gersh BJ, McGoon MD, et al. Long-term outcome after surgical repair of isolated atrial septal defect. Follow-up at 27 to 32 years. N Engl J Med 1990;323(24):1645-50.

6. Veldtman GR, Razack $\vee$, Siu S, et al. Right ventricular form and function after percutaneous atrial septal defect device closure. J Am Coll Cardiol 2001;37(8):2108-13.

7. Akagi T. Current concept of transcatheter closure of atrial septal defect in adults. J Cardiol 2015;65(1):17-25.

8. Rosenhek R, Gabriel H, Rader F, et al. Benefit of atrial septal defect closure in adults: impact of age. Eur Heart J 201 1;32(5):553-60.

9. Brochu M-C, Baril J-F, Dore A, et al. Improvement in exercise capacity in asymptomatic and mildly symptomatic adults after atrial septal defect percutaneous closure. Circulation 2002;106(14):1821-6.

10. Giardini A, Donti A, Specchia S, et al. Recovery kinetics of oxygen uptake is prolonged in adults with an atrial septal defect and improves after transcatheter closure. Am Heart J 2004;147(5):910-4.

11. Giardini A, Donti A, Formigari R, et al. Determinants of cardiopulmonary functional improvement after transcatheter atrial septal defect closure in asymptomatic adults. J Am Coll Cardiol 2004;43(10):1886-91.

12. Warnes CA, Williams RG, Bashore TM, et al. ACC/AHA 2008 Guidelines for the Management of Adults with Congenital Heart Disease: a report of the American College of Cardiology/American Heart Association Task Force on Practice Guidelines. Circulation 2008; 1 18(23):e71 4-833

13. Baumgartner $\mathrm{H}$, Bonhoeffer $\mathrm{P}, \mathrm{De}$ Groot NMS, et al. ESC Guidelines for the management of grown-up congenital heart disease (new version 2010). Eur Heart J 2010;31(23):2915-57. 
14. Cowley CG, Lloyd TR, Bove EL, et al. Comparison of results of closure of secundum atrial septal defect by surgery versus Amplatzer septal occluder. Am J Cardiol 2001;88(5):589-91.

15. Du ZD, Hijazi ZM, Kleinman CS, et al. Comparison between transcatheter and surgical closure of secundum atrial septal defect in children and adults: results of a multicenter nonrandomized trial. J Am Coll Cardiol 2002;39(11):1836-44.

16. Oto A, Ozkutlu S, Canpolat U, et al. Transcatheter interatrial septal defect closure in a large cohort: midterm follow-up results. Congenit Heart Dis 2013;8(5):418-27.

17. Silversides CK, Haberer K, Siu SC, et al. Predictors of atrial arrhythmias after device closure of secundum type atrial septal defects in adults. Am J Cardiol 2008;101(5):683-7.

18. Butera $G$, Carminati $M$, Chessa $M$, et al. Percutaneous versus surgical closure of secundum atrial septal defect: comparison of early results and complications. Am Heart J 2006;151(1):228-34.

19. Krumsdorf U, Ostermayer S, Billinger K, et al. Incidence and clinical course of thrombus formation on atrial septal defect and patient foramen ovale closure devices in 1,000 consecutive patients. J Am Coll Cardiol 2004;43(2):302-9

20. Durongpisitkul K, Chanthong P, Chungsomprasong P, et al. Beneficial effects of transcatheter closure of atrial septal defects not only in young adults. J Intervent Cardiol 2012;25(4):382-90.

21. Zahn E, Khan D. Mid-term outcomes of the Helex septal occluder for percutaneous closure of secundum atrial septal defects. Congenit Heart Dis 2013;8(5):428-33.

22. Kutty S, Hazeem AA, Brown K, et al. Long-term (5- to 20-year) outcomes after transcatheter or surgical treatment of hemodynamically significant isolated secundum atrial septal defect. Am J Cardiol 2012;109(9): 1348-52.

23. Dell'Avvocata F, Cardaioli P, Giordan M, et al. Five-year follow-up of transcatheter intracardiac echocardiography-assisted closure of interatrial shunts. Cardiovasc Revasc Med $2011 ; 12(6): 355-61$.

24. Li Y, Zhou K, Hua Y, et al. Amplatzer occluder versus CardioSEAL/ STARFlex occluder: a meta-analysis of the efficacy and safety of transcatheter occlusion for patent foramen ovale and atrial septal defect. Cardiol Young 2013;23(4):582-96.
25. Taaffe M, Fischer E, Baranowski A, et al. Comparison of three patent foramen ovale closure devices in a randomized trial (Amplatzer versus CardioSEAL-STARflex versus Helex occluder). Am J Cardiol 2008;101(9):1353-8.

26. Luermans JG, Post MC, ten Berg JM, et al. Long-term outcome of percutaneous closure of secundum-type atrial septal defects in adults. Eurolntervention 2010;6(5):604-10.

27. Staubach S, Steinberg DH, Zimmermann W, et al. New onset atrial fibrillation after patent foramen ovale closure. Catheter Cardiovasc Interv 2009;74(6):889-95.

28. Abdi $S$, Kiani R, Momtahen $M$, et al. Percutaneous device closure for secundum-type atrial septal defect: short and intermediate-term results. Arch Iran Med 2012;15(11):693-5.

29. Fischer G, Stieh J, Uebing A, et al. Experience with transcatheter closure of secundum atrial septal defects using the Amplatzer septal occluder: a single centre study in 236 consecutive patients. Heart 2003;89(2): 199-204.

30. Walters DL, Boga T, Burstow D, et al. Percutaneous ASD closure in a large Australian series: short- and long-term outcomes. Heart Lung Circ 2012;21(9):572-5

31. Rocchini AP, Lloyd TR, Aiyagari RM. Long-term follow up of secundum atrial septal defect closure with the amplatzer septal occluder. Congenit Heart Dis 2010;5(1):32-7.

32. Kazmi T, Rehman AU, Latif $F$, et al. Device closure of atrial septal defect: medium-term outcome with special reference to complications. Cardiol Young 2012;22(1):71-8.

33. Butera $G$, Carminati $M$, Chessa $M$, et al. CardioSEAL/STARflex versus Amplatzer devices for percutaneous closure of small to moderate (up to $18 \mathrm{~mm}$ ) atrial septal defects. Am Heart J 2004; 148(3):507-10.

34. Kotowycz MA, Therrien J, lonescu-ltu R, et al. Long-term outcomes after surgical versus transcatheter closure of atrial septal defects in adults. JACC Cardiovasc Interv 2013;6(5):497-503.

35. Ninios VN, Mezilis NE, Tsikaderis DD, et al. Percutaneous closure of atrial septal defects: immediate and mid-term results. J Cardiol 2010;51(2):104-12. 